\title{
ULTRASOUND-GUIDED PERIPHERAL NERVE BLOCKS, A SAFETY METHOD OF ANESTHESIA IN PATIENTS WITH SEPSIS WITH AN INITIAL ACUTE RESPIRATORY FAILURE - PRESENTATION OF TWO CLINICAL CASES
}

\author{
Petya Ivanova $^{1}$, Nikolay Mladenov ${ }^{1}$, Deyan Anakievski ${ }^{2}$, Viliyan Platikanov ${ }^{1}$ \\ ${ }^{1}$ Clinic of Anesthesiology and Intensive Care, St. Marina University Hospital, Varna, \\ Medical University of Varna \\ ${ }^{2}$ Clinic of Urology, St. Marina University Hospital, Varna, Medical University of Varna
}

\begin{abstract}
Anesthetic management of patients with severe sepsis is a great challenge. Systemic inflammation and acute organ dysfunction in response to infection is a major problem, especially respiratory failure and hemodynamic instability. Avoidance of lung injury during mechanical ventilation is possible with peripheral nerve blocks.

Clinical case 1: We present a 73-year-old male hemodialysis patient with sepsis. He had infectious complication of aneurysm formation of $\mathrm{A}-\mathrm{V}$ fistula. The patient was hypoxic - $\mathrm{SpO}_{2} 86-88 \%$, with presence of tachypnea, RR-150/75, HR-125/min, Temp $-38^{0} \mathrm{C}$, coagulation abnormalities - INR 1, 58 (clopidogrel intake), elevated CRP and WBC.

The patient was indicated for emergency procedures of incision, drainage and ligation of A-V fistula. We performed supraclavicular brachial plexus block + sedation.

Clinical case 2: We present a 61-year-old woman with sepsis, with past medical history of diabetes, COPD, and endometrial cancer. She was with clinical presentation of necrotizing fasciitis of the upper extremity.

We performed ultrasound-guided supraclavicular brachial plexus block - "in plane" technique, $30 \mathrm{~mL} / 25$ $\mathrm{mL}$ ropivacaine $0.5 \%$ in moderate sedated patients.

During the operation the patients were conscious, hemodynamically and respiratory stable, with oxygen supply by a mask, and with excellent intraoperative and postoperative pain control.

We think that ultrasound-guided peripheral nerve blocks are safe and effective alternatives for septic patients with/without coagulation abnormalities.
\end{abstract}

Keywords: US-guided peripheral nerve blocks, sepsis

\footnotetext{
Address for correspondence:

Petya Ivanova

St. Marina University Hospital

1 Hristo Smirnenski Blvd

9000 Varna

e-mail:pivanova82@gmail.com
}

Received: August 23, 2019

Accepted: September 6, 2019

\section{INTRODUCTION}

The management of a septic patient continues to be a major challenge, both in resuscitation and anesthesiology aspects, despite the enormous advances of medicine in this area in recent years. Systemic inflammatory response and acute organ dysfunction are the main problems, particularly acute respiratory failure and hemodynamic instability, which an an- 
esthesiologist faces in such patients on the operating table. When the source of sepsis is a surgically solvable problem, any delay and postponement of surgical intervention to stabilize organ systems is not the best solution. Each patient has a unique clinical situation, not a recurrent phenomenon with a clear clinical outcome. Is it not more appropriate to postpone mechanical ventilation when possible and to expect a favorable outcome after surgery and powerful broad spectrum antibiotic therapy? Or is this just postponing the inevitable? Are peripheral nerve blocks a sure and safe method of anesthesia and postoperative analgesia in these clinical situations? Would they provide optimal analgesia in compromised acidosis conditions? These are questions where the responses are dependent on subjective factors - clinical knowledge, experience, attitude, and not on objective factors. The categorical advantages offered by regional anesthesia in this situation are avoidance of: 1 . general anesthesia and mechanical ventilation; 2 . high doses of opioids in the first 24 hours, which would lead, along with general anesthesia, to a higher incidence of cognitive impairment, as well as better local rheological conditions (1-3).

\section{AIM}

The aim of this article is to present two clinical cases of septic patients with respiratory failure in which surgical intervention to resolve the infectious source has occurred under ultrasound(US)-guided supraclavicular block.

\section{CASE PRESENTATION}

We present two clinical cases: patients with sepsis and acute myocardial infarction undergoing operative upper limb interventions to resolve the infectious source.

\section{Clinical Case 1:}

We present a 73-year-old man with co-morbidity chronic kidney disease (CKD) - chroniodialysis and ischemic heart disease with a clinical picture of sepsis resulting from an infected aneurysmal A-V fistula. Clinical signs: adynamics, somnolence, tachypnea 25-30/min, hypoxic $\mathrm{SpO}_{2} 84-86 \%$ on atmospheric air, $\mathrm{O}_{2}$ mask $6 \mathrm{~L} / \mathrm{min} \mathrm{SpO}_{2}$ 92-94\%, RR - 150/75, HR - 125/min C. Laboratory constellation: CRP - $190 \mathrm{mg} / \mathrm{L}, \mathrm{WBC}-19$. The patient was also with an abnormal coagulation status of INR -
1.58 , with oral administration of clopidogrel and aspirin protect.

The surgical intervention was performed in the following order: after an $\mathrm{O} 2$ mask of $6 \mathrm{~L} / \mathrm{min}$, the patient was monitored for ECG, $\mathrm{SpO}_{2}$. Following premedication of $1 \mathrm{mg}$ midazolam and $50 \mu \mathrm{g}$ of fentanyl, a US-guided supraclavicular block was performed with $30 \mathrm{~mL}$ ropivacaine $0.5 \%$ - $150 \mathrm{mg}$. The surgical intervention started 30 minutes after the local anesthetic was instilled, and once again $1 \mathrm{mg}$ midazolam $+50 \mu \mathrm{g}$ fentanyl were administered before the surgical incision. An incision was made, about $30 \mathrm{~mL}$ of purulent content were evacuated, followed by drainage and ligation of the A-V fistula. The operative intervention was performed smoothly with excellent surgical and postoperative pain control, hemodynamically stable, with no respiratory changes.

\section{Clinical Case 2:}

We present a 61-year-old woman with a severe upper limb infection, necrotizing fasciitis, with a 4-day prescription, covering a wrist area of $7-8 \mathrm{~cm}$ below the shoulder joint. There was severe underlying pathology consisting of endometrial carcinoma with subsequent chemotherapy, obesity - BW $100 \mathrm{~kg}$, ID - type 2, COPD, and ischemic heart disease. She was with a clinical picture of sepsis - somnolent to unresponsive in some moments, sometimes confused. There were limb lesions with the affected limb being very painful, swollen, and presence of hyperemia with necrosis and destruction of tissue in the area of the forearm and abundant purulent secretion. The clinical signs were: RS - tachypnea 30/ min, blood gas sample - hypoxemia with hypocapnia. $\mathrm{SpO}_{2}-88 \%$ at room air with $\mathrm{O}_{2}$-mask 6-7 $\mathrm{L} / \mathrm{min}$, $\mathrm{SpO}_{2}$ 96-97\%. RR - 140/90, HR - 90/min, temperature $35.8^{\circ} \mathrm{C}$. Laboratory performance: CRP -390 $\mathrm{mg} / \mathrm{L}, \mathrm{WBC}-22$, blood glucose - $20 \mathrm{mmol} / \mathrm{L}$. The patient was monitored - ECG, pulse oximetry, and $\mathrm{O}_{2}$ mask was placed.

After fluid resuspension 500 mL Hartmann + $250 \mathrm{~mL}$ colloidal solution, the patient was premedicated with $50 \mu \mathrm{g}$ of fentanyl. Supraplavicular block - "in plane" ropivacaine $125 \mathrm{mg}$ - $25 \mathrm{~mL}, 0.5 \%$ was performed. Drainage, evacuation of necrotic materials and fasciotomy took place. The patient remained hemodynamically stable with no change in the respi- 
Petya Ivanova, Nikolay Mladenov, Deyan Anakievski et al.

ratory pattern. Intraoperatively, 2 x $25 \mathrm{mg}$ of calypsol was administrated.

\section{RESULTS}

The intraoperative period for both patients was smooth, with no hemodynamic and respiratory problems, and optimal intra- and post-operative analgesia.

\section{Clinical case 1:}

The patient was placed in a clinic for vascular surgery with $\mathrm{O}_{2}$ therapy for the first 24 hours. He received a broad-spectrum antibiotic combination. After $48 \mathrm{~h}$ he was subfebrile at times, after 72 hours afebrile. There was no tachypnea and need of $\mathrm{O}_{2}$ after the 24th hour. He was discharged on the 7th postoperative day.

\section{Clinical case 2:}

The patient entered into resuscitation with $\mathrm{O}_{2}$ therapy and broad-spectrum antibiotic combination: meropenem + vancomycin + metronidazole. There was a decrease in the inflammation markers, CRP was $72 \mathrm{mg} / \mathrm{L}$ on the 4 th postoperative day, but with cognitive disturbances. On the 6th postoperative day, she was admitted to an endocrinology clinic and on the 14th postoperative day she was discharged by the medical establishment. On the 5th and 9th postoperative day, the early issues were treated with short, moderate sedation.

\section{CONCLUSION}

We and other authors believe that US-guided peripheral nerve blocks are a safe and reliable method in some cases of septic patients with an initial respiratory failure, as well as in patients with abnormal coagulation status.

\section{REFERENCES}

1. Eissa D, Carton EG, Buggy DJ. Anaesthetic management of patients with severe sepsis. Br J Anaesth. 2010;105(6):734-43. doi: 10.1093/bja/aeq305.

2. Fatungase OM Ogundipe AA Adebanjo AA. Usefulness of peripheral nerve block as an aneasthetic technique in a critically ill child - a case report. $\mathrm{Ni}$ ger J Paediatr. 2017; 44(1):39-41.

3. Kumar TS, Indu K, Parthasarathy S. Successful management of above knee amputation with combined and modified nerve blocks. Anesth Essays Res. 2017;11(2):520-51. doi:10.4103/0259-1162.183161. 